\title{
Dynamic routing discovery scheme for high mobility in mobile ad hoc wireless networks
}

\author{
Haider Alani ${ }^{1}$, Maha Abdelhaq ${ }^{2}$, Raed Alsaqour ${ }^{3}$ \\ ${ }^{1}$ IT Center and System, Iraqi Ministry of Electricity, Baghdad, Iraq \\ ${ }^{2}$ Department of Information Technology, College of Computer and Information Sciences, \\ Princess Nourah bint Abdulrahman University, Saudi Arabia \\ ${ }^{3}$ Department of Information Technology, College of Computing and Informatics, \\ Saudi Electronic University, Saudi Arabia
}

\begin{tabular}{l}
\hline Article Info \\
\hline Article history: \\
Received Dec 18, 2019 \\
Revised Jan 29, 2020 \\
Accepted Feb 1, 2020 \\
\hline
\end{tabular}

Keywords:

AODV

Link breakage

MANET

Mobility

Routing discovery

\begin{abstract}
An innovative technology that is widely used in many applications is the Mobile Ad-hoc Network (MANET). Discovery and maintenance of routes at MANET are important issues. Within MANET, broadcasting is used to discover a path within on-demand routing protocols. Establishing and maintaining a route periodically among the nodes is the challenge that requires the transmitting of control packets across a network. This state leads to the issue of broadcasting storms. Broadcasting control packets increase control packets overhead and decrease network performance. In this paper, we proposed a scheme called AODV-Velocity and Dynamic (AODV-VD) for effective broadcast control packets. The routing protocol for the ad-hoc on-demand distance victor (AODV) is used to implement the proposed AODV-VD scheme. AODV-VD scheme reduces both the excessive route discovery control packets and network overhead. Network simulator version 2.35 (NS2.35) was used to compare the proposed AODV-VD scheme to the AODV routing protocol in terms of end-to-end latency, average throughput, packet transmission ratio and overhead ratio.
\end{abstract}

Copyright $\odot 2020$ Institute of Advanced Engineering and Science. All rights reserved.

\section{Corresponding Author:}

Maha Abdelhaq,

Department of Information Technology,

College of Computer and Information Sciences,

Princess Nourah bint Abdulrahman University,

84428 Riyadh, Saudi Arabia.

Email: MSAbdelhaq@pnu.edu.sa, maha.ukm@gmail.com

\section{INTRODUCTION}

Mobile Ad-hoc Network (MANET) is a mobile, infrastructure-free, self-configuring, interactive and multi-hop network [1]. MANET is an emergency network with a range of mobile nodes. The nodes in MANET move randomly within the network due to rapid and frequent topology changes in the MANET [2]. Such mobile nodes can function simultaneously as hosts and routers since they can travel anywhere in the MANET [3]. In MANET, it is the responsibility of the routing protocol to develop a route between nodes to transmit packets, which selects the optimum and maintains the route. When a source node wants to transmit data, the routing protocol constructs a path between a source node and a destination node. MANET routing protocols are divided into three classifications: positive, reactive and hybrid [4].

In MANET routing protocols, a broadcasting scheme is necessary when packets are sent between mobile nodes to maintain network connectivity [5]. Broadcasting is usually defined as the process of transmitting a packet to all nodes in the network from a source node. Broadcasting is more frequently used in MANET when the source node broadcasts Route Request (RREQ) packets to check for a route to a destination node, usually in path discovery. Broadcasting is often used in route maintenance when nodes 
exchange Hello packets for gathering neighboring information. Intermediate nodes in MANET assist in the broadcast process. Intermediate nodes are responsible for forwarding the packet to other nodes in the network from the source node [6].

The flooding results in multiple redundant transmissions on the network, in which a node from various nodes can get the payload of the same packet. This event is known as a broadcasting storm problem [7]. The issue of broadcasting storms leads to regular contention and packet collisions, which increases network overhead traffic. In the route maintenance phase, the broadcast storm problem occurs, during which routes are updated by activating new route discovery requests to replace broken routes. In this paper, we suggested a scheme called AODV-Velocity and Dynamic (AODV-VD) for efficient broadcast control packets. The results obtained from evaluating the AODV-VD scheme are very positive and show the effectiveness of the proposed scheme in mitigating the problem of the broadcast storm.

The remainder of the paper is structured according to this. We provide a description of the research background and work related to it in Section 2. Section 3 describes the new scheme AODV-VD. Section 4 describes the environmental simulation and the performance metric. Section 5 addresses the findings and evaluations and Section 6 summarizes our research and our future work.

\section{BACKGROUND AND RELATED WORK}

\subsection{Ad-hoc on-demand distance vector routing protocol}

AODV, that Perkins and Royer built [8], is a reactive routing protocol, and its multi-hop routing protocol and discovery react on demand. In the AODV routing protocol, the strengths of Destination Sequenced Distance Vector Routing (DSDV) and Dynamic Source Routing (DSR) protocols are combined [8]. The source node broadcasts a route request (RREQ) packet throughout the MANET nodes in the route discovery process of the AODV routing protocol on MANET and sets a timer to wait for the reply. The RREQ packet contains routing information, including the IP address of originator, ID of broadcast and sequence number of destination.

Each intermediate node receives the RREQ packet and holds two operations running the reverse path to the source node. First, the intermediate node checks if it has previously received the RREQ packet with the same originator IP address and broadcast ID, and then determine whether the RREQ packet should be rejected or accepted. Second, if the RREQ packet is accepted the intermediate node will check the destination sequence number stored in its routing table. The intermediate node uni-cast the Route Reply (RREP) packet to the source node if the number of the sequence is greater than or equal to the number contained in the RREQ. If no intermediate node has a sufficiently fresh path to the destination node (new destination sequence number), then the RREQ packet must continue navigating until it reaches the destination node. Figure 1 shows the source node $(\mathrm{S})$ that transmits RREQ packets over the network to its neighboring nodes until the RREQ packet reaches the destination node (D). Figure 2 also shows the destination node (D) which replies to the source node with an RREP.

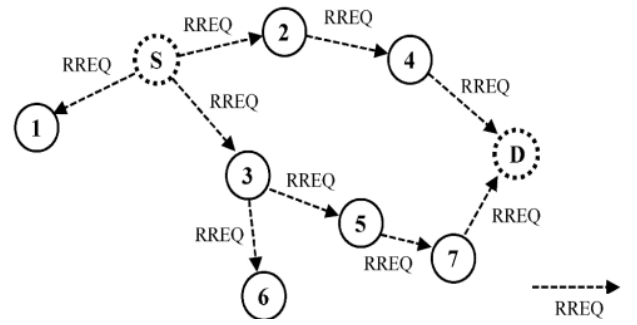

Figure 1. AODV broadcasts RREQ packet

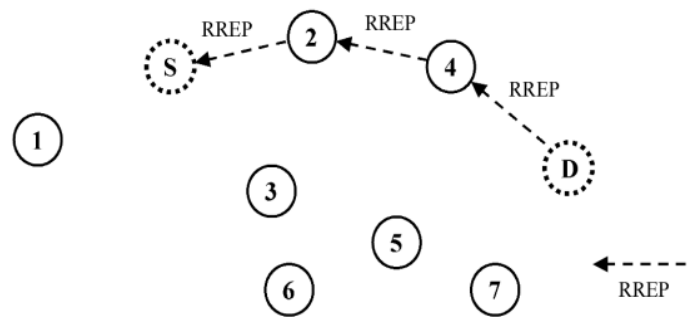

Figure 2. AODV replies RREP packet

In AODV, every node in the network also sends Hello packet periodically to keep its routing table at the one-hop neighbor. A Hello packet is used to determine if the neighboring connection is still alive. The node sends Hello packet with a time interval called hello-interval to its neighbor node to detect broken links between the nodes. Every node sends Hello packets to its neighbors and they receive their acknowledgment. If a node sends Hello packets to a neighbor twice and has not received a message of acknowledgment for it, then the node initiates the broken link process.

\subsection{Related work}

In [9], the authors proposed a routing protocol called Mobility Aware and Dual-Phase Ad-hoc On-demand Distance Vector with Adaptive Hello Messages (MA-DP-AODV-AHM). It focuses primarily on

Dynamic routing discovery scheme for high mobility in mobile ad hoc wireless networks (Haider Alani) 
developing routes that consider the speeds and direction of motion of vehicles with regard to source vehicles, thereby providing more efficient routes and reducing route breaks. The authors have suggested an adaptive packet notification system, which is directly connected to the regular hello messages solution, with the speeds of the vehicles, resulting in a substantial reduction in the amount of overhead control and network congestion. The protocol was evaluated using QualNet simulator in terms of overhead control, end-to-end delay and energy consumption. Results of the simulation summarized that MA-DP-AODV-AHM effectively contributes to mitigating network instability by producing reliable, secure routes and reducing connection failures. It has also shown its superiority over AODV and MDA-AODV.

In [10], the authors introduced a Quality of Service (QoS) algorithm in ad hoc networks. The proposed algorithm entitled Modified Light Weight distributed the QoS Algorithm, taking into account the position and relative velocity of the nodes and estimating the importance of linking reliability, choosing a path with longer duration, reducing link breaks and enhancing QoS. The authors measured the algorithm's efficiency in packet delivery ratio, packet delivery latency, average end-to-end delay overhead routing and packets lost. The simulation results show that the Modified Light Weight distributed QoS Algorithm enhances the QoS parameters of QoS in ad hoc networks.

In [11], the authors proposed two predictive link availability techniques performed during the control packet routing. The first technique referred to as zone-based estimation divides each node's area of transmission into three areas, namely inner, middle, and outer, based on the signal strength obtained. Here, nodes lying in the inner and outer zone are prohibited from transmitting the control packet as they have extremely high and low signal strength received, respectively. Nodes in the middle region are only permitted inside the locality for the control packet flow. The second technique is an improvement over zone-based estimation, in which nodes are considered for route selection in the outer zone and are referred to as segment-based estimation. It calculates the link availability ratio of the node for each adjacent link based on the neighbor's current position, its adjacent segment, and the sector in which the neighbor is present within the node's transmit region. Such information assists in the transfer of control packets within the outer region. The simulation was performed over DSR and AODV protocols in terms of control overhead, bandwidth, number of control packets, length of the route, and number of route errors generated within the network. The results show that knowledge of the link status during the routing phase helps to find more stable routes with reduced overhead control and thus increases the overall performance of the network.

In [12], a neighboring information-based broadcasting scheme is being introduced to minimize latency for ad hoc wireless networks. In the scheme, few Hello messages are interchanged to gather information about one-hop neighbors. The information collected is used to calculate the density of the neighbor, the ratio, and the number of exposed neighbors with one-hop, on which the probability and delay of retransmission are modified. The way the probability and delay of rebroadcasting are described in the neighboring knowledge-based broadcast scheme decreases the overhead transmission and effectively restrains the aggregation of traffic. After that, a method for the velocity-based delivery of data is proposed and applied to a neighboring knowledge-based broadcast scheme to further reduce latency, creating neighboring knowledge and speed-based broadcast scheme. It is specified that few higher-velocity nodes are used to retransmit the incoming message with a greater probability. The simulation determines the schemes efficiency under different network configurations. The results show that the new scheme outperforms current broadcast schemes in terms of overhead, and in particular, in the average end-to-end delay. The proposed scheme lowers the overhead by 88.4 percent relative to flooding and the average end-to-end delay by a maximum of 88.9 percent.

In [13], the authors suggested a novel discovery scheme within MANET called the Dynamic Probabilistic Route (DPR). The DPR scheme forwarded the packets with a dynamically determined probability known as a forwarding probability (FP) to the neighboring node. The likelihood of forwarding is dynamically determined to depend on two factors; the first is the density of neighboring local nodes and the second is the total number of its neighbors protected by broadcast. Using the DPR scheme, network performance is higher than other protocols.

In [14], the authors introduced a new probabilistic broadcasting scheme for MANETs that overcomes the constraints of existing broadcasting schemes. Through detailed simulations, it is shown that the newly proposed schemes in different operating conditions and scenarios outperform previous broadcast schemes. Unlike previous works, the proposed broadcast scheme strategy is based on information from the node velocity vector to adjust the retransmission probability and categorize the reliability of the nodes accordingly. The authors applied this velocity vector and evaluated its efficiency with respect to various important metrics such as link stability and overhead RREQ packets. The key benefit of this scheme is to avoid the traditional AODV's route re-discovery process, especially at high mobility nodes.

A novel scheme for Channel Adaptive Probabilistic Broadcast (CAPB) is proposed in [15], which is implemented in the AODV routing protocol to replace pure flood-based broadcast. Under the scheme, 
the probability of retransmitting RREQ packets is regulated by the current signal interference plus noise ratio (SINR) and neighboring density of the nodes. Compared to the other three broadcasting schemes which include AODV, fixed probabilistic scheme AODV-P, and the dynamic noise-dependent probabilistic scheme (DNDP), the CAPB scheme performs better in terms of overhead routing, throughput and end-to-end delay.

Energy Efficient Neighbor Coverage protocol (EENCP) was proposed by [16] to route discovery in MANET. Uncovered neighbor and neighborhood node density are two metric factors considered under the EENCP protocol to reduce the forwarding of the RREQ packet. The forwarding of the RREQ packet by the node relies on its battery life and neighborhood density, which forwards when there is adequate energy and when the node density surpasses a particular threshold. The EENCP protocol can limit rebroadcasting with packets more than AODV and Neighbor Coverage-based Probabilistic Rebroadcast (NCPR) protocols. A new probabilistic vector velocity scheme was developed in [14] for route discovery in MANET. Based on the AODV routing protocol, this scheme categorizes all mobile nodes into reliable nodes and unreliable nodes in terms of sender and receiver node velocity, and assigns a high retransmission probability for reliable nodes and a low retransmission probability for unreliable nodes. This type of scheme helps to discover the steadiest and most efficient routes, thereby enhancing route discovery efficiency. This scheme demonstrates the RREQ packet's superiority in terms of both overhead and connection stability. A novel algorithm, Reliability Factor-based Routing Protocol (RFBRP), was introduced by [17] to discover a reliable route in MANET. It is designed based on reactive routing protocols such as AODV and DSR. It is positioned on the reliability factor (RF) between the intermediate nodes to select a dependable route. RF is a primary metric that limits the routing failure and the number of route discovery requests, reducing the overhead costs of each node in the route discovery process. In terms of overhead, delivery of a packet, and end-to-end delay, the RFBRP algorithm performs better than AODV and semi-proactive AODV (SP-AODV) protocols.

Hanji and Shettar [18] introduced an Improved AODV (I-AODV) protocol in MANET. The route discovery in the I-AODV protocol was based on the location and energy of the nodes in the network. The source node selects the intermediate node located in the communication range, which has less distance than the other intermediate nodes to the destination node and has energy greater than the other intermediate nodes to increase the lifetime of the path. The I-AODV protocol provides efficient packet transmission, eliminates overhead and improves the lifetime of the path that leads to a stable path compared to the AODV protocol.

Kok, et al. [19] proposed an effective ad-hoc wireless broadcasting scheme, known as Improved Partial Dominant Pruning (IPDP), based on Partial Dominant Pruning (PDP). It uses neighborhood information to reduce redundancy of broadcasting and ensuring that all nodes in a network are reachable to packets. To compare their efficiency in two sample sets, IPDP and PDP were built. The first sample in static scenarios while the second set of data in mobile scenarios examines the schemes. The IPDP is very effective in static scenarios in reducing the redundancy of broadcast while maintaining availability to all nodes in a network. From the mobile scenario, all variants of IPDP performed at low to high node movement speed close PDP in terms of reachability. A new AODV (NAODV) in MANET was suggested by [20] in the routing protocol. It resolves the AODV routing protocol issues by accounting local congestion of the network while selecting the route. Based on network congestion, the number of hops is identified while selecting the next node to limit the survival time of forward and reverse routes, and reduce the number of RREQ packet retransmissions, reducing overhead costs. Mohsin, et al. [21] examine the effects of the density and mobility nodes of different maritime traffic model based on the capabilities of three MANET routing protocols that comprise AODV, AOMDV and DSDV. Various node densities and mobility interpret the traffic models that will be mainly found in the marine environment. The performance assessment of the MANET protocols is judged in terms of packet delivery ratio.

Zohra and Rahman [22] introduced the dynamic probabilistic broadcast algorithm in MANET. This algorithm estimates the rebroadcast probability by utilizing the forwarding probability of a node. The algorithm introduced a logical model to identify the expected number of forwarding nodes that are essential to accomplish a broadcast in a self-pruning algorithm. It employs neighbor knowledge to limit the unnecessary retransmissions in MANET. It exhibits superior performance compared to the static probabilistic algorithm and the probabilistic adjust broadcast algorithm. A dynamic partitioning scheme (DPS) dependent on nodal vehicles was suggested by Rayeni, et al. [23]. The DPS model computes partition sizing and quantities, after which it computes a size and broadcast timetable for each partition. DPS scheme performs reliably and time-efficiently in an emergency broadcast program across multiple hops in either low or high-density traffic states. This method suitably applies to varied scenarios featuring scarce, mid and high-density traffic. Simulations confirm that DPS performs outstandingly with low latencies and high reliability. Kimura, et al. [24] presented a density-aware probabilistic routing model featuring adaptive probabilistic forwarding operation, wherein the forwarding probabilities decline as messages are redirected. Such density-sensitive probabilistic routing models control the speeds of circulating copies of messages in 
high-density areas. The results of the performance assessment present the adaptive probabilistic forwarding model in a high-density nodal region. The On-demand, Tree-based Routing Protocol (OTRP) was suggested by Al Aamri, et al. [25] for MANET. The OTRP with AODV protocol allowed it to scale up and also lessen overheads in MANET routing discovery, by pairing the concept of hop-to-hop routed transmissions with the routing discovery algorithms known as Tree-based Optimized Flooding (TOF). The primary elements which govern OTRP performance were hypothetically examined and assessed in terms of nodal branch counts and locations, and counts of RREQ routing expirations. At various levels of nodal density and mobility, OTRP was contrasted to AODV, OLSR, and dynamic MANET On-demand (DYMO) routing protocols. The findings indicate that OTRP mechanisms perform outstandingly with much-lessened network overhead and declining traffic loads.

Zhang, et al. [26] suggested Neighboring Coverage-derived Probabilistic Rebroadcasting (NCPR) protocols for MANET. The method dynamically tallies rebroadcasting delays and rebroadcasting probabilities. Rebroadcasting delays are used to resolve the forwarding orders and to efficiently utilize neighboring coverage information to derive further and more exact coverage ratios. Connection factors are employed in NCPR to determine rebroadcasting probabilities, to maintain networked connectivity, and to decrease incidences of redundantly retransmitted traffic. Research on routing requests in routing discovery methods contrasted NCPR with Dynamically Probabilistic Route discovery protocols (DPR), and AODV protocols. NCPR generates less traffic rebroadcasts than flooding protocols as a consequence of fewer redundantly rebroadcast traffic, which decreases incidences of packet collisions and network contention, raises the proportion of packet deliveries, and diminishes average end-to-end latencies.

\section{PROPOSED AODV VELOCITY AND DYNAMIC (AODV-VD) SCHEME}

The design and implementation of the proposed AODV-VD algorithm is based on the literature proposed Advance Velocity Aware Probabilistic (AVAP)-AODV scheme by [14], AODV-VD scheme makes use of probabilistic velocity vector to initiate route discovery. Each mobile node is classified as either a Reliable Node (RN) or an UnReliable Node (URN). This classification is based on the sender and receiver nodes' velocity vector. Reliable nodes are assigned a high rebroadcast probability value, while unreliable nodes are given a low rebroadcast probability value. The scheme proposed by Kanakaris, et al. [27], is considered a dynamic density-driven route request forwarding scheme. Moreover, this made it, possible for each node to use its neighborhood density as a basis for forwarding the RREQ message. The neighborhood's node density plays a vital role in determining the rebroadcast probability [15].

The proposed AODV-VD scheme transmits an RREQ packet while discovering a route between the nodes, AODV-VD scheme uses the node's dynamic density and velocity vector. It aims to reduce the unnecessary broadcasting of route control packets and identify the reliable intermediate nodes existing between the source and destination nodes. AODV-VD scheme makes use of several adjustable parameters to determine whether retransmission is permitted or forbidden. The information obtained from the node density within the neighborhood can be utilized to rebroadcast RREQ, selectively. If the neighbourhood has many nodes, then the aim would be to decrease the probability of retransmission to avoid possible redundancy. In this case, nodes that are only one hop away are defined as being "adjacent". Therefore, if a node sends an RREQ packet, retransmission can only be performed by a subset of nodes in its neighborhood. The number of nodes allowed to perform retransmission can vary dynamically depending on the neighborhood node. Table 1 shows the parameters used in the AODV-VD scheme. The AODV-VD scheme flowchart can be seen in Figure 3 When any node $(F i=1,2,3, . ., n)$ receives the RREQ packet, the proposed AODV-VD scheme can be performed and the packet is processed as follows Figure 3.

Table 1. AODV-VD parameters specification

\begin{tabular}{ll}
\hline Parameters & Description \\
\hline$n$ & total number of nodes presented in the network \\
$F i$ & set of nodes that have received the RREQ message \\
$i$ & range from 1 to n number of nodes $\eta$ \\
$\beta_{i}$ & number of nodes adjacent to node i \\
$P_{i}$ & packet forwarding probability based on $\beta \mathrm{i}$ \\
$d$ & node adjacency \\
$C_{f}$ & control factor between $(0,1)$ adjusted using Pi \\
$R$ & random number in the range $[0,100]$ \\
$\theta t h$ & predefined angle threshold \\
$\theta$ & Cosine angle between transmitter and receiver to \\
& decide if the receiver is RN or URN \\
\hline
\end{tabular}

Int J Elec \& Comp Eng, Vol. 10, No. 4, August 2020 : 3702 - 3714 


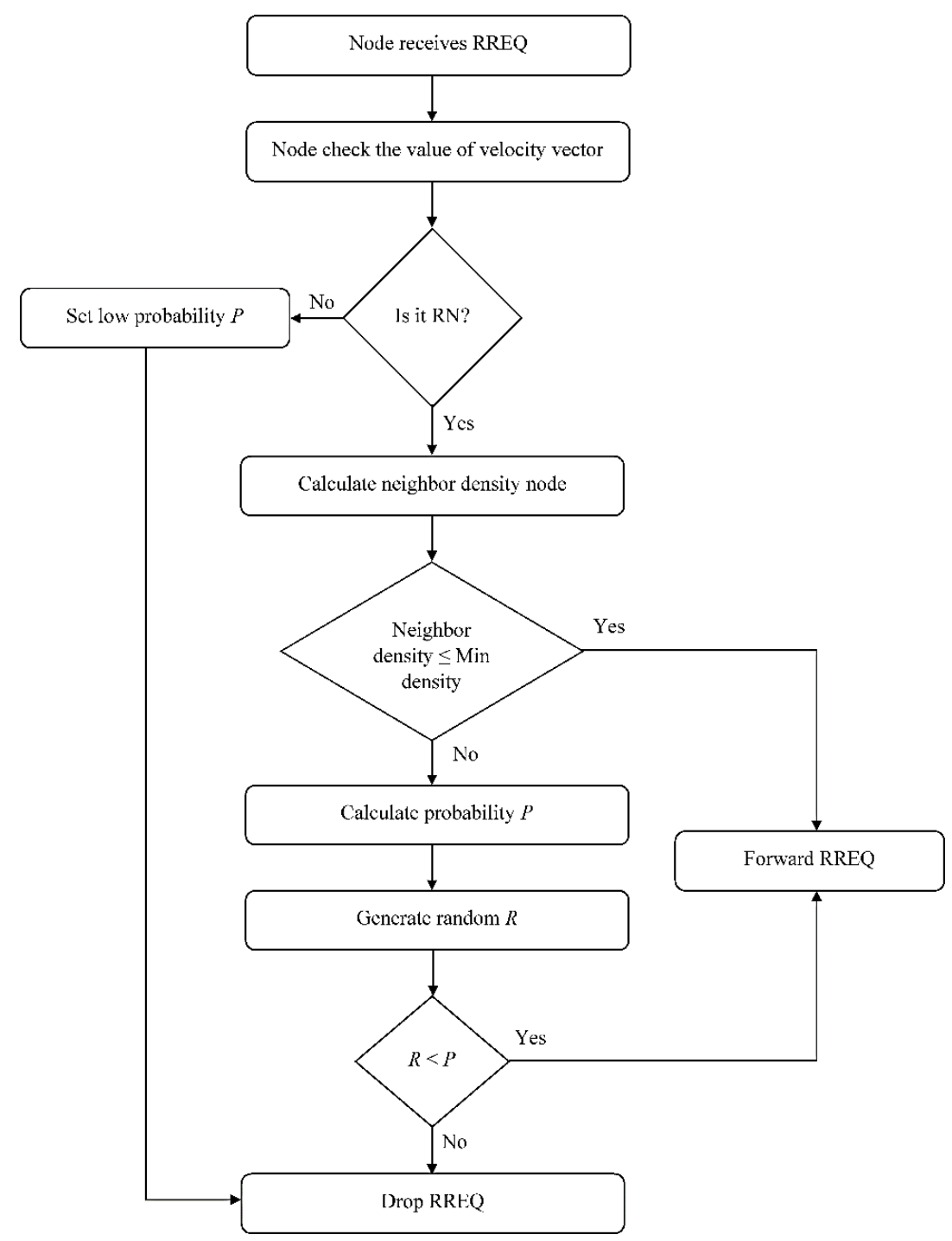

Figure 3. AODV-VD scheme flowchart

1. When a source node (S) tries to discover a new route, its velocity vector is placed in the RREQ packet header.

2. When this RREQ packet is obtained by any node receiver $(R)$ within the broadcast range of $S$, the cosine angle $\theta$ is calculated. It then makes a rebroadcast decision using the following process:

- If the node's cosine angle value is $\theta>\theta$ th, then the receiver node is categorized as a URN. It then assigns a low rebroadcast probability.

- If the node's cosine angle value is $\theta<\theta$ th, then the receiver node is categorized as $\mathrm{RN}$. Then go to STEP 3.

3. If $\beta \mathrm{i} \leq \mathrm{d}$ then else

Forward the RREQ packet

Calculate forwarding probability $\mathrm{Pi}$ at node $\mathrm{Fi}$

$$
\mathrm{Pi}=\frac{100}{\beta \mathrm{i}} *\left(\mathrm{~d} * \mathrm{C}_{\mathrm{f}} ; \quad \text { For } 0<\mathrm{C}_{\mathrm{f}} \leq 1\right.
$$

4. Generation of a Random Number $(\mathrm{R})$ between $(0,100)$

5. If $\mathrm{R}<\mathrm{Pi}$ then else

Forward the RREQ packet

6. End

Ignore and Drop the RREQ packet 
The proposed AODV-VD scheme for MANET aims to improve the network performance and resolve the issue of the frequent link breakage. The scheme selects the reliable node during the route discovery process to avoid the link break and eliminate redundant retransmission to achieve the lowest value of congestion, therefore, reduce the overhead in the network [28].

\section{SIMULATION ENVIRONMENT}

We used NS-2.35 to measure the AODV-VD scheme's performance. The nodes are moving according to the random waypoint mobility model. In the random waypoint mobility model, nodes move freely and randomly without border restriction in this mobility model. CBR traffic is generated by the application layer at the node. For every node, the transmission range is $250 \mathrm{~m}$. The pause time for the node is constant at 0 to allow node movement at all times. Network scenario node speed was selected at $5,10,20,30,40$, and $50 \mathrm{~m} / \mathrm{s}$, and all scenarios set the number of nodes at 50 . All nodes are set in a $1000 \mathrm{~m}$ square area of about $1000 \mathrm{~m}$. Table 2 displays the parameters in the simulation.

Table 2. Simulation parameters

\begin{tabular}{lll}
\hline Parameters & Value & Unit \\
\hline Simulation time & 300 & $\mathrm{~S}$ \\
Network area size & $1000 \times 1000$ & $\mathrm{M}$ \\
Number of nodes & $20-100$ & Nodes \\
Data packet size & 512 & Bytes \\
Bandwidth & 2 & $\mathrm{Mbps}$ \\
Nodes speed & $5-50$ & $\mathrm{~m} / \mathrm{s}$ \\
Pause time & 0 & $\mathrm{~S}$ \\
Data traffic & $\mathrm{CBR}$ & \\
\hline
\end{tabular}

Performance metrics are used in the comparative study to assess the performance of the proposed AODV-VD scheme. The following criteria are used for evaluating the proposed AODV-VD scheme in scenarios.

a. Average end-to-end (E2E) delay

The average end-to-end delay metric is the average time it takes to successfully relay the data packet from the source through the network to destination. This delay includes many smaller network delays, including all potential delays caused by buffering latency during route discovery, queuing at router interface queue, delays in MAC retransmission, propagation and transmission time. The average data packet delay for the E2E can be determined using the following formula:

$$
\text { Avg E2E delay }=\frac{\sum_{i-1}^{n}\left(R_{i}-S_{i}\right)}{n}
$$

where $R i$ is the total packet received, $S i$ is the total packet sent, and $n$ is the number of data packets.

b. Average throughput (Avg-Throughput)

The average throughput metric is the average of received successful data packets to the total simulation time period. The total throughput is expressed in kilobits per second (kbps), and measures the routing protocol's efficiency and reliability in receiving data packets through destinations. The formula used to calculate the average throughput is as follows:

$$
\text { Average Throughput }=\frac{\sum \text { Packets received by destinations }}{\text { stop time-start time }} * \frac{8}{1000}
$$

c. Packet delivery ratio (PDR)

The PDR metric shows the total number of received data packets by destinations divided by the total number of data packets sent by the sources. This metric presents how a protocol successfully delivers packets from the source to the destination. A high packet delivery ratio indicates good results, which represent the wholeness and correctness of the routing protocol. The packet delivery ratio is computed using the following formula:

$$
P D R=\frac{\text { Dpackets received by destinations }}{\sum \text { packets sent by sources }} * 100
$$




\section{d. Packet overhead ratio (POR)}

Routing overhead ratio metric is the ratio of the total number of routing packets sent to the total number of routing packets sent and the data packets sent. This metric gives an idea about the extra bandwidth the overhead uses to handle data traffic. The overhead routing is calculated using formulae below:

$$
R O R=\frac{\text { No of routing packets }}{\text { No of routing packets }+ \text { No of data packets sent }} * 100
$$

\section{e. Average of collision rate (ACR)}

The ACR signifies the total number of RREQ packets that have been dropped and which did not successfully arrive at the nodes during the simulation period. The formula below shows how the ACR is calculated:

$$
A C R(\text { packet } / \mathrm{sec})=\frac{\sum \text { Number of } R R E Q \text { packet failed }}{\text { simulation time }}
$$

\section{f. Link stability}

Link stability pertains to the total number of broken routes that are found during data transmission between nodes during the simulation time. Route break measurements are done despite varying network densities and node speeds. Calculation of the link stability follows the following formula:

$$
\text { Link stability (link/route) = total number of route breakage }
$$

\section{RESULTS AND DISCUSSIONS}

\subsection{Results based on the number of nodes}

The network density is considered to be a significant and decisive parameter. The number of nodes under different network densities was set to 20,40,60, 80, and 100 during the simulation, and for all the network parameters, every node was set to a maximum speed of $20 \mathrm{~m} / \mathrm{s}$. Figure 4 shows the average throughput versus the number of nodes. Figure 4 shows there is a small decrease in the average throughput of the proposed AODV-VD scheme when the number of nodes is 40 compared to the modified AVAP-AODV and the original AODV protocols. In a dense network, the network becomes more stable. Therefore, there is an increase in the average throughput in a dense network and convergent ratio in different protocols. Figure 4 also shows that the AODV-VD has experienced a standard increase in average throughput with the decrease in intensity of dropping nodes. This can be due to the random deployment of nodes that change following its number in a network.

Figure 5 demonstrates the varying average delay of E2E by contrasting the current AODV-VD scheme with the initial AODV protocols and AVAP-AODV. The figure shows a considerable difference between those protocols in the average E2E delay. Compared to the original AODV and AVAP-AODV protocols, the average E2E latency in the proposed AODV-VD is reduced. For example, in a dense network with 100 nodes, the average E2E delay for AODV, AVAP-AODV, and AODV-VD is 0.11898s, 0.079727s, and $0.0640505 \mathrm{~s}$, respectively. This might be the cause behind the decrease in packet drop over the network. This occurs because the dense network, the routes between source and destination nodes is a stable route. And the stable route yields to lesser route breakages and lesser re-establishment that take less delay time and an increase in the average throughput, as observed in Figure 4.

The POR results shown in Figure 6 imply that the proposed AODV-VD scheme has enabled a reduction of POR necessary to allocate the routes in various sources and destinations, followed by the AVAP-AODV and original AODV protocols. The control factor in the feeding network with prior knowledge of the node adjacency dropped in different nodes has played a significant role in the reduction of the POR. As observed in Figure 6 in the high-density area, there is an increase in POR because of having many routes leading to the generation of more control packets. AODV-VD technique, in the proposed scheme, generates fewer control packets.

Figure 7 illustrates the effects of the different protocols on the ACR comparison. The results indicate that when moving from a low-density region to a high-density area, the ACR increases dramatically. This is because as the number of nodes increases, so does the number of possible forwarded nodes. When the number of nodes increased progressively, the proposed AODV-VD scheme led to less collision. The result has established the efficiency of dynamic density-driven route requests to decrease the collision by supporting a peer-to-peer connection between terminals. But AVAP-AODV and the original AODV appeared to pose approximately similar ACR, which is greater than the proposed AODV-VD. Additionally, allowing the lowest amount of ACR in the network would result in less power consumption. 
Figure 8 shows that the proposed AODV-VD led to a reduced number of link breakages compared to the AVAP-AODV and the AODV protocols. The number of broken links decreases when the number of nodes increases because the network has the propensity to be stable in a dense network. As observed in Figure 8, the AODV-VD scheme has lesser broken links in different nodes densities, due to the technique used to select the reliable neighbor node. This study clearly shows that offering a protocol to reduce the probability of route breakage would enable enhancement of the route efficiency, which helps to consume less power and generates fewer control packets used in the network.

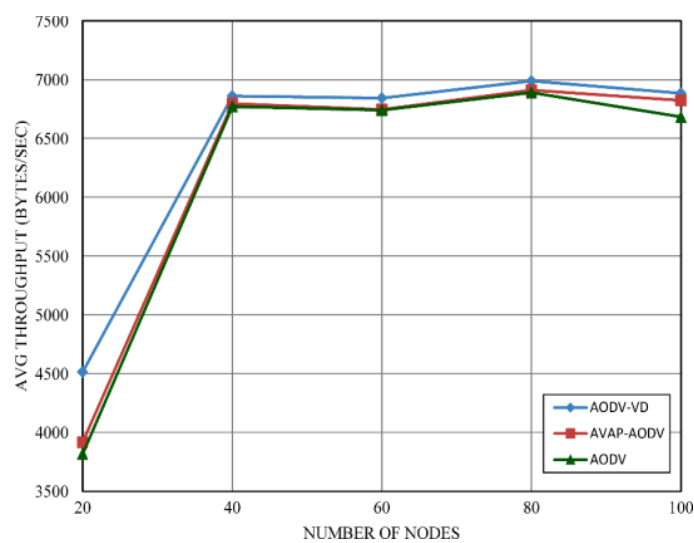

Figure 4. Avg throughput vs number of nodes

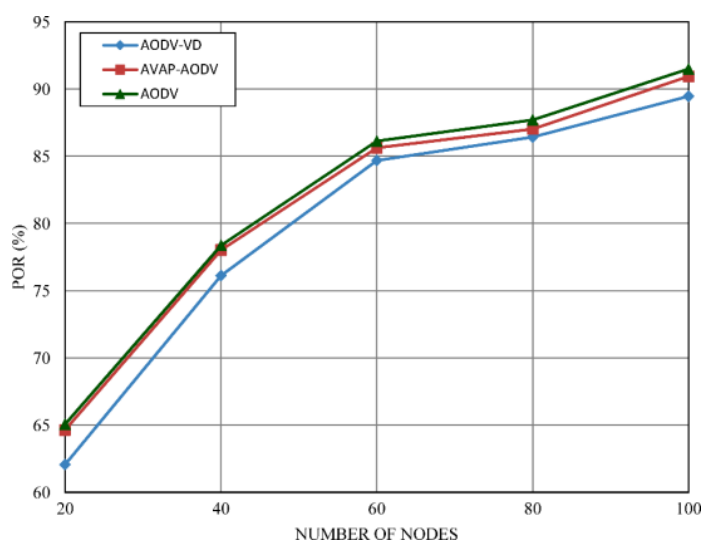

Figure 6. POR vs number of nodes

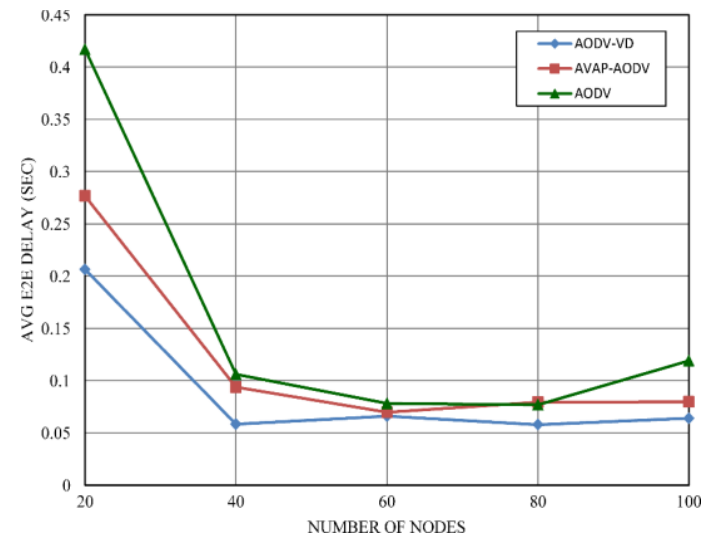

Figure 5. Avg E2E delay vs number of nodes

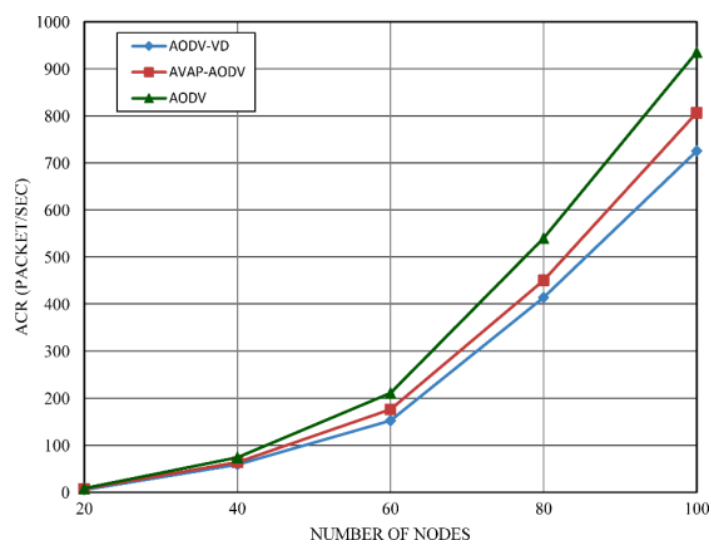

Figure 7. ACR vs number of nodes

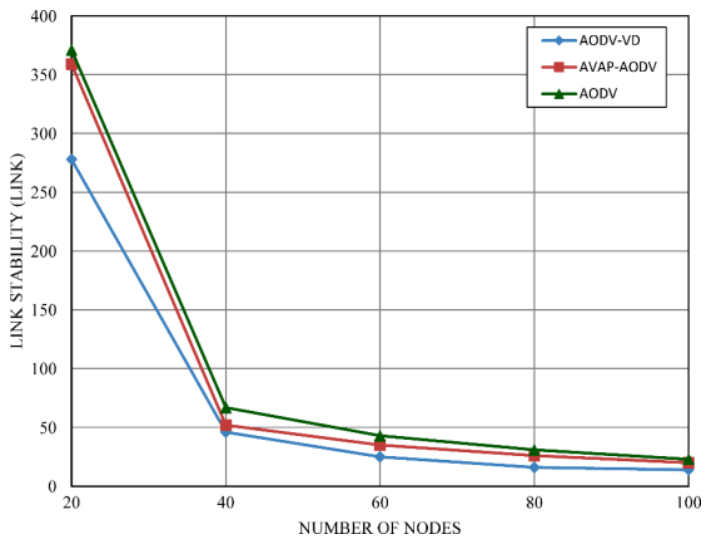

Figure 8 . Link stability vs the number of nodes 


\subsection{Results based on the speed of nodes}

Figure 9 shows the varying average throughput by comparing the proposed AODV-VD scheme with the original AODV and the AVAP-AODV protocols. Figure 9 shows that the proposed scheme has appreciably outpaced both AVAP-AODV and the earlier AODV, as evidenced in the data, particularly as node speed ratios rise progressively. This is due to the proposed scheme selecting the reliable node and lesser controls packet leading to the transfer of more data packets.

Figure 10 illustrates the average E2E delay of packet data transfers for the routing discovery protocol, according to the various node speeds. As node speed increases, the average E2E delay of packet data transfers increases proportionately. This occurs because as node speed increases along the routes between source and destination nodes, recurrent breakages and re-establishment in the link occur. Results for the AODV-VD scheme show that it yields considerably fewer delays in comparison to AVAP-AODV and the earlier AODV.

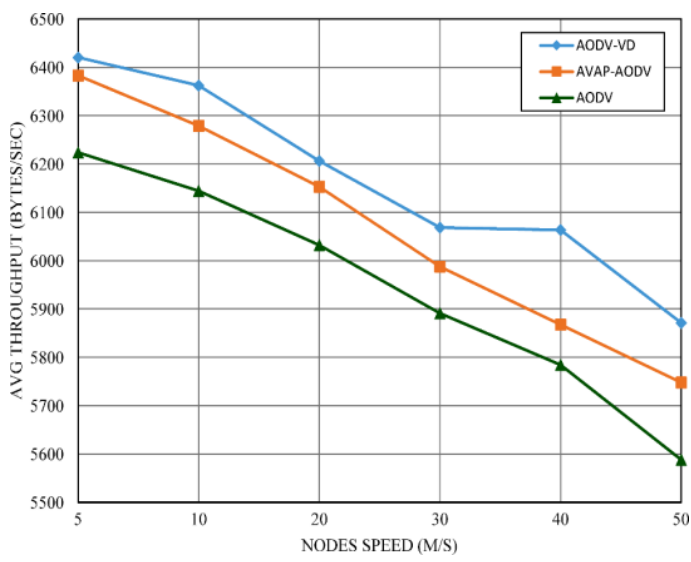

Figure 9. Avg throughput vs speed of nodes

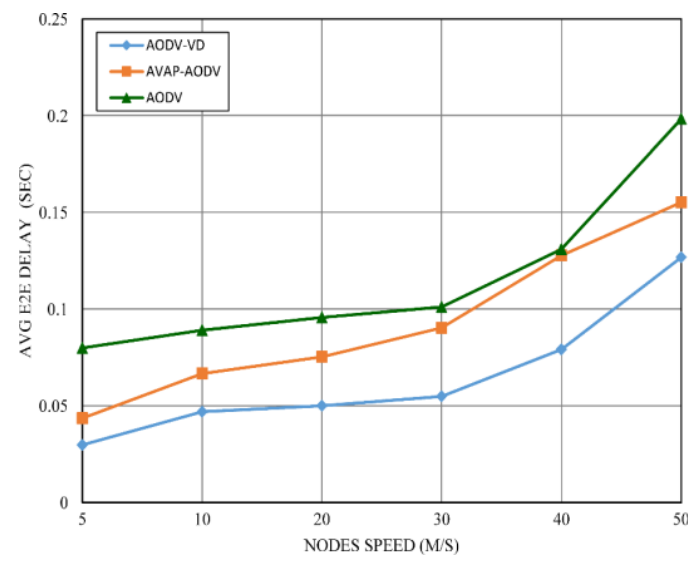

Figure 10. Avg E2E delay vs speed of nodes

Figure 11 shows the three protocols in comparison according to the POR and the different speeds. The acquired results indicated a significant disparity in POR, showing its lowest peaks in those cases where the proposed AODV-VD scheme was running at different speed ranges. Compared with that of AVAP-AODV and the earlier AODV, the new scheme provided fewer POR. This is due to the AODV-VD scheme's ability to control local area densities while RREQ is selectively rebroadcast.

Figure 12 shows comparative results for the ACR of packet transfers according to the node speeds. The results acquired reveal that the earlier AODV protocol generated higher rates of collisions, followed by the AVAP-AODV protocol. The least value for ACR was recorded when the proposed AODV-VD scheme was executed at various speeds. This is attributable to the efficiency of the AODV-VD mechanism in determining the state for decreasing the nodes in the neighborhood, especially in the event of high probability conditions. The lower number of collisions in the network helps to consume less power.

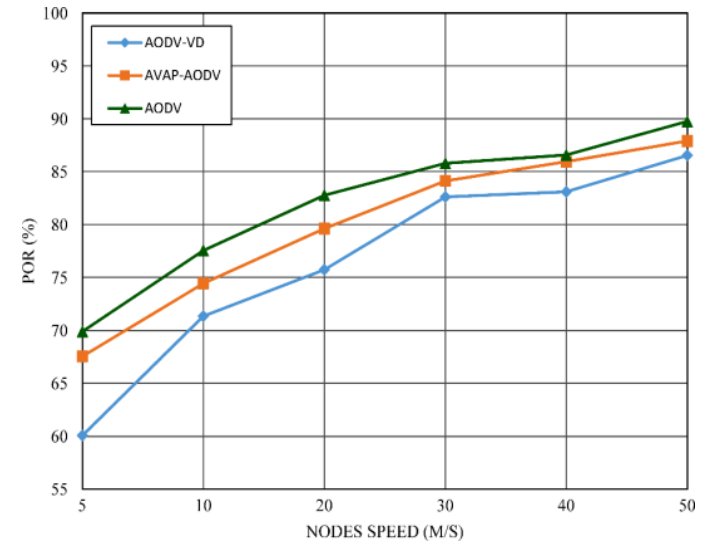

Figure 11. POR vs speed of nodes

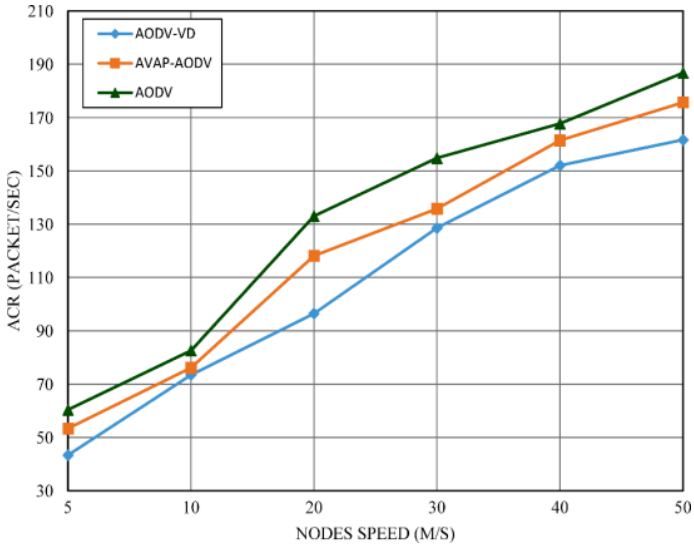

Figure 12. ACR vs speed of nodes 
Figure 13 illustrates the comparative results for linkage stability, which represents the number of route breakages according to the various node speeds, which ensued in the course of the simulations. As node speeds increase, the number of route breakages correspondingly increases, since, during high-speed operation, nodes run paths to route breakage during their occurrences. Notably, the proposed AODV-VD scheme generates fewer instances of route breakage in comparison to the two other protocols.

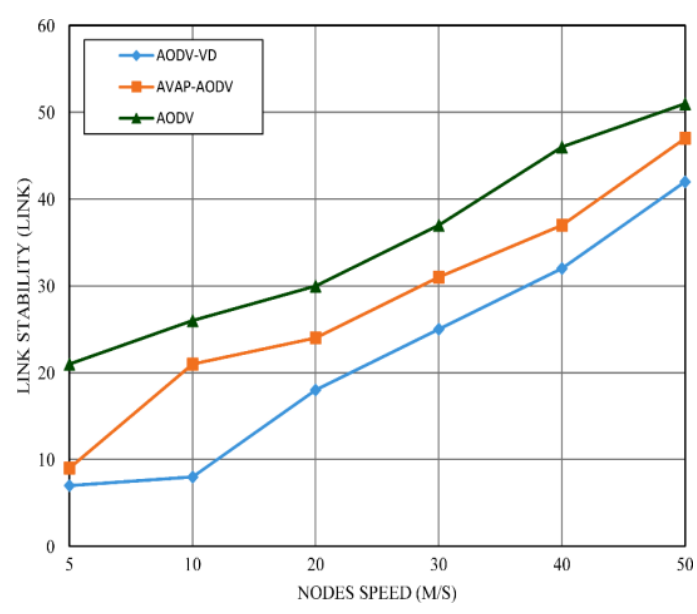

Figure 13. Link stability vs speed of nodes

\section{CONCLUSION AND FUTURE WORK}

This paper is focused on proposing the newer dynamically probabilistic route discovery scheme for MANET. The scheme aims to improve network performance and resolve the problem of frequent link breakage. The scheme selects the reliable node of the route discovery process to avoid the link break and eliminate redundant retransmission to achieve the lowest value of congestion then, therefore, reduce the overhead in the network. AODV-VD scheme initializes probabilistic density values when nodes receive RREQ packets corresponding to globalized and localized information on node neighbor densities. Exponential math functions are utilized in the technique to resolve probability values for every node, dynamically. Thus, the scheme can be useful for reducing retransmission activity involving redundant packets. The proposed AODV-VD scheme was compared with the original AODV and AVAP-AODV protocols. The protocols were compared based on their performance to two sets of disparate node speeds and various numbers of nodes. The first set evaluates the protocols in various numbers of nodes, the number of nodes under different network densities was set to 20,40,60, 80, and 100 during the simulation, and for all the network parameters, every node was set to a maximum speed of $20 \mathrm{~m} / \mathrm{s}$. The second set evaluates the protocols according to the corresponding nodal speeds of $5 \mathrm{~m} / \mathrm{s}, 10 \mathrm{~m} / \mathrm{s}, 20 \mathrm{~m} / \mathrm{s}, 30 \mathrm{~m} / \mathrm{s}, 40 \mathrm{~m} / \mathrm{s}$, and $50 \mathrm{~m} / \mathrm{s}$ during the simulation, the network density was set 50 nodes. The simulation outcomes indicate that the AODV-VD scheme performs outstandingly. In this research, the proposed scheme performance was assessed with the aid of current AODV protocols under NS2.35. It would be appropriate for additional reactive protocol types such as DSR to be included in an operational assessment of the proposed schemes performance. Additionally, the performance of the proposed scheme can be analysed with the use of VANET. Finally, the random way mobility model was used to evaluate the performance of the AODV-VD scheme. Several different mobility models can be used to evaluate the performance of the AODV-VD scheme in a different model that simulates another environment.

\section{ACKNOWLEDGMENT}

This research was funded by the Deanship of Scientific Research at Princess Nourah bint Abdulrahman University through the Fast-track Research Funding Program.

\section{REFERENCES}

[1] C. Reddy, "Node activity based trust and reputation estimation approach for secure and QoS routing in MANET," International Journal of Electrical \& Computer Engineering (IJECE), vol. 9, no. 6, pp. 5340-5350, 2019. 
[2] M.S. Abdelhaq, R.A. Alsaqour, M. Al-Hubaishi, T. Alahdal, and M. Uddin, "The Impact of Resource Consumption Attack on Mobile Ad-hoc Network Routing," IJ Network Security, vol. 16, pp. 376-381, 2014.

[3] A. Srivastava, A. Mishra, B. Upadhyay, and A.K. Yadav, "Survey and overview of Mobile Ad-Hoc Network routing protocols," in 2014 International Conference on Advances in Engineering and Technology Research (ICAETR), pp. 1-6, 2014.

[4] R. Mylsamy and S. Sankaranarayanan, "A Preference-Based Protocol for Trust and Head Selection for ClusterBased MANET," Wireless Personal Communications, vol. 86, pp. 1611-1627, 2016.

[5] M. B. Yassein, M. B. Khalaf, and A. Y. Al-Dubai, "A new probabilistic broadcasting scheme for mobile ad hoc ondemand distance vector (AODV) routed networks," The Journal of Supercomputing, vol. 53, pp. 196-211, 2010.

[6] D. K. Panda and R. K. Dash, "Reliability Evaluation and Analysis of Mobile Ad Hoc Networks," International Journal of Electrical \& Computer Engineering (IJECE), vol. 7, no. 1, pp. 479-485, 2017.

[7] Y.-C. Tseng, S.-Y. Ni, Y.-S. Chen, and J.-P. Sheu, "The broadcast storm problem in a mobile ad hoc network," Wireless networks, vol. 8, pp. 153-167, 2002.

[8] C. Perkins, E. Belding-Royer, S. Das, "Ad hoc on-demand distance vector (AODV) routing," RFC Editor, 2003.

[9] K. A. Darabkh, M. S. Judeh, H. B. Salameh, and S. Althunibat, "Mobility aware and dual phase AODV protocol with adaptive hello messages over vehicular ad hoc networks," AEU-International Journal of Electronics and Communications, vol. 94, pp. 277-292, 2018.

[10] S. H. Hosseini Nazhad Ghazani, "Modified Light Weight distributed QoS Algorithm for wide area ad hoc networks," International Journal of Communication Systems, vol. 31, pp. e3562, 2018.

[11] S. R. Malwe, N. Taneja, and G. Biswas, "Enhancement of DSR and AODV Protocols Using Link Availability Prediction," Wireless Personal Communications, vol. 97, pp. 4451-4466, 2017.

[12] D. Lu and S. Dong, "A neighbor knowledge and velocity-based broadcast scheme for wireless ad hoc networks," International Journal of Distributed Sensor Networks, vol. 13, no. 11, pp. 1-14, 2017.

[13] K. Shanmugam, K. Subburathinam, and A. Velayuthampalayam Palanisamy, "A Dynamic Probabilistic Based Broadcasting Scheme for MANETs," The Scientific World Journal, vol. 2016, 2016.

[14] M. B. Khalaf, A. Y. Al-Dubai, and G. Min, "New efficient velocity-aware probabilistic route discovery schemes for high mobility Ad hoc networks," Journal of Computer and System Sciences, vol. 81, pp. 97-109, 2015.

[15] H. Y. Adarbah, S. Ahmad, B. Arafeh, and A. Duffy, "Efficient broadcasting for route discovery in mobile ad-hoc networks," in Proceedings of the International Symposium on Performance Evaluation of Computer and Telecommunication Systems, pp. 1-7, 2015.

[16] R. R. Ravi and V. Jayanthi, "Energy Efficient Neighbor Coverage Protocol for Reducing Rebroadcast in MANET," Procedia Computer Science, vol. 47, pp. 417-423, 2015.

[17] S. M. Khan, R. Nilavalan, and A. F. Sallama, "A Novel Approach for Reliable Route Discovery in Mobile Ad-Hoc Network," Wireless Personal Communications, vol. 83, pp. 1519-1529, 2015.

[18] B. R. Hanji and R. Shettar, "Improved AODV with restricted route discovery area," in 2015 International Conference on Computer Communication and Informatics (ICCCI), pp. 1-6, 2015.

[19] G.-X. Kok, C.-O. Chow, and H. Ishii, "Reducing Broadcast Redundancy in Wireless Ad-Hoc Networks with Implicit Coordination Among Forwarding Nodes," Wireless Personal Communications, vol. 81, pp. 253-278, 2015.

[20] Z. Zhao, M. Gao, M. Hou, and N. Zhang, "Design of Redundant New Ad-Hoc On-Demand Distance Vector (NAODV) Routing Protocol Based on Congestion and Survival Control," Wireless Personal Communications, vol. 85 , pp. $2657-2668,2015$.

[21] R. J. Mohsin, J. Woods, and M. Q. Shawkat, "Density and mobility impact on MANET routing protocols in a maritime environment," in Science and Information Conference (SAI) 2015, pp. 1046-1051, 2015.

[22] F. T. Zohra and A. Rahman, "Mathematical analysis of self-pruning and a new dynamic probabilistic broadcast for MANETs," in 2015 International Conference on Networking Systems and Security (NSysS), pp. 1-9, 2015.

[23] M.S. Rayeni, A. Hafid, and P.K. Sahu, "Dynamic spatial partition density-based emergency message dissemination in VANETs," Vehicular Communications, vol. 2, pp. 208-222, 2015.

[24] T. Kimura, T. Jonouchi, T. Matsuda, and T. Takine, "Density-aware store-carry-forward routing with adaptive forwarding probability control," in 2015 IEEE International Conference on Consumer Electronics-Taiwan (ICCE-TW), pp. 474-475, 2015.

[25] H. AlAamri, M. Abolhasan, D. Franklin, and J. Lipman, "Optimised relay selection for route discovery in reactive routing," Ad Hoc Networks, vol. 11, pp. 70-88, 2013.

[26] X.M. Zhang, E.B. Wang, J.J. Xia, and D.K. Sung, "A neighbor coverage-based probabilistic rebroadcast for reducing routing overhead in mobile ad hoc networks," Mobile Computing, IEEE Transactions on, vol. 12, pp. 424-433, 2013.

[27] V. Kanakaris, D. Ndzi, and K. Ovaliadis, "Improving AODV performance using dynamic density driven route request forwarding," arXiv preprint arXi, 1107.3630, 2011.

[28] S. Sharma, D. Jindal, and R. Agarwal, "Analysing Mobile Random Early Detection for Congestion Control in Mobile Ad-hoc Network," International Journal of Electrical \& Computer Engineering (IJECE), vol. 8, no. 3, pp. 1305-1314, 2018. 


\section{BIOGRAPHIES OF AUTHORS}

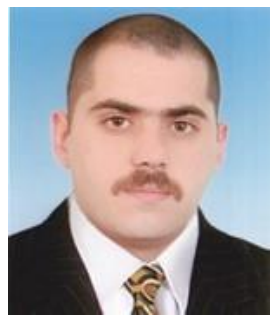

Haider Alani is a Programmer at the IT Center in the Ministry of Electricity of the Republic of Iraq, Baghdad, Iraq. He received his BSc degree in Computer Science from Alrafidain University College (RUC) Iraq, in 2004. MSc degree in Computer Science from Universiti Kebangsaan Malaysia (UKM) in 2016. His research interests include mobile ad hoc network (MANET) and vehicular ad hoc network (VANET).

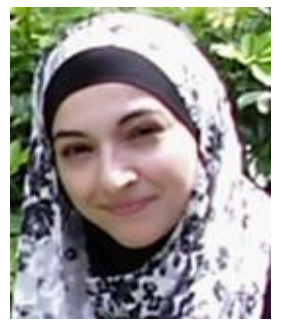

Maha Abdelhaq is an Assistant Professor at College of Computer and Information Sciences, Princess Nourah bint Abdulrahman University, Saudi Arabia. She received her PhD from the Faculty of Information Science and Technology, National University of Malaysia, Malaysia in 2014; MSc in securing wireless communications from the University of Jordan, Jordan in 2009; BSc in Computer Science from the University of Jordan, Jordan in 2006. She is a member in IEEE, ACM and International Association of Engineers (IAENG). Her research interests include vehicular networks, MANET routing protocols, artificial immune systems, network security and intelligent computational.

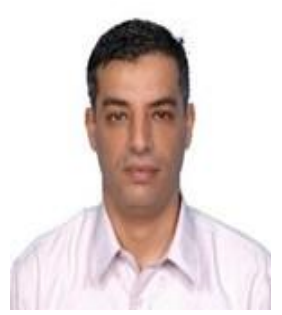

Raed Alsaqour is an Associate Professor at College of Computation and Informatics, Saudi Electronic University, Riyadh Branch, Kingdom of Saudi Arabia. He received his $\mathrm{PhD}$ in Wireless Communication Systems from National University of Malaysia, Malaysia in 2008; MSc in Distributed Systems from University Putra Malaysia, Malaysia in 2003; BSc in Computer Science from Mutah University, Jordan in 1997. He is a member in IEEE, ACM and IAENG. His research interests include wireless ad hoc and vehicular networks, routing protocols, simulation and network performance evaluation. 